\title{
The Safety and Feasibility of Low-Molecular-Weight Heparin Prophylaxis in Major Abdominal Surgery Combined with Neuraxial Anesthesia
}

\author{
Bogdan Protyniak ${ }^{1}$, Michael C. Meadows ${ }^{1,2}$, H. Rae Pak ${ }^{3}$, Ronald S. Chamberlain ${ }^{1,2,4}$ \\ ${ }^{1}$ School of Medicine, Saint George’s University, Saint George, Grenada \\ ${ }^{2}$ Department of Surgery, Saint Barnabas Medical Center, Livingston, USA \\ ${ }^{3}$ Department of Anesthesia, Saint Barnabas Medical Center, Livingston, USA \\ ${ }^{4}$ Department of Surgery, University of Medicine and Dentistry of New Jersey, Newark, USA \\ Email: rchamberlain@sbhcs.com
}

Received May 8, 2011; revised November 11, 2011; accepted December 2, 2011

\begin{abstract}
Background: Global guidelines for venous thromboembolism (VTE) prophylaxis of patients undergoing major surgery are well established. However, their applicability and safety in patients receiving neuraxial anesthesia is unproven. We sought to evaluate the safety and feasibility of chemical VTE prophylaxis in a prospective group of patients undergoing major foregut procedures under a combination of epidural and general anesthesia. Methods: A prospective database of all patients undergoing major foregut surgery from 2004-2009 was maintained and analyzed. Epidural catheters were placed pre-operatively and used for post-operative analgesia for three days in all patients. Factors evaluated included age, ethnicity, sex, length of stay, duration of epidural placement, complications of epidural placement and post-operative management, and VTE events. A uniform protocol was followed regarding the timing of low-molecular-weight heparin (LMWH) administration with epidural catheter insertion/removal. Results: A total of 237 patients formed the study group. The mean age was 57 years (range, 19 - 88) among 121 (51.1\%) women and 65 years (range, 20 - 95) among 116 (48.9\%) men. One hundred and sixty-six patients were Caucasian (70\%), 37 Black (15.6\%), 15 Hispanic (6.3\%), 12 Asian/Pacific (5.1\%), and 7 other (3\%). All epidural catheters were removed on the third post-operative day. There were a total of five VTE (2.1\%) events postoperatively. No peri-operative or post-operative epidural catheter associated complications occurred. Conclusions: Concomitant epidural catheterization and LMWH anticoagulation is safe and feasible in major abdominal surgery patients, including those undergoing major hepatic resection. Guidelines for VTE prophylaxis and LMWH administration in the setting of neuraxial anesthesia are well established and applicable to this unique patient population.
\end{abstract}

Keywords: Abdominal Surgery; Epidural Anesthesia; Low-Molecular-Weight Heparin; Complications, Venous Thromboembolism

\section{Introduction}

In recent years, general anesthesia in combination with neuraxial anesthesia has become widely used in orthopedic, cardiovascular, and abdominal surgery [1-3]. Epidural anesthesia offers many advantages when compared to the use of general anesthesia combined with systemic opioids for post-operative pain relief. These include superior pain control, decreased stress response, lowered risk of thromboembolism, and improved immune function [4-7].

The patient's coagulation status and the physical act of removing the catheter are critical factors which contribute to the development of spinal hematoma, resulting in neurologic complications [8]. Patients undergoing he- patic surgery are at especially high risk for bleeding due to anticipated postoperative coagulation profile derangements, marked by decreased platelet count and increased PT and a PTT [9,10]. As a result, some clinicians have refrained from the use of epidural analgesia in conventional hepatic surgery as well as liver transplant surgery [11,12].

Numerous studies have documented the safety of anticoagulation with neuraxial anesthesia in orthopedic, cardiovascular, and obstetric surgery $[1,2,13]$. To date, however, very few anesthesiologists or surgeons have investigated the safety and feasibility of this combination under a uniform protocol in major abdominal surgery, despite the fact that patients undergoing major foregut surgery, such as hepatectomy, pancreatectomy, or sple- 
nectomy, represent a high-risk venous thromboembolic disease (VTE) group [14]. This study reviews the safety and feasibility of anticoagulation with neuraxial anesthesia in a prospective group of patients undergoing major abdominal surgery.

\section{Methods}

A total of 237 patients formed the study group. The mean age was 57 years (range, 19 - 88) among 121 (51.1\%) women and 65 years (range, 20 - 95) among 116 (48.9\%) men. One hundred and sixty-six patients were Caucasian (70\%), 37 Black (15.6\%), 15 Hispanic (6.3\%), 12 Asian/ Pacific (5.1\%), and 7 other (3\%) (Table 1).

Data was collected on all patients undergoing major foregut surgery by a single surgeon from 2004-2009 after IRB approval. Preoperative epidural catheters were placed in the thoracic space (T8-T10) in all patients. Thirty minutes prior to surgery, a dose of $0.1 \%$ bupivicaine and fentanyl $2 \mu \mathrm{g} / \mathrm{mL}$ was diluted in $5 \mathrm{~mL}$ of normal saline and administered through the catheter at a rate of $6-8$ $\mathrm{mL} / \mathrm{hr}$. General anesthesia was provided using isoflurane, fentanyl, and cisatracurium as a muscle relaxant. Additional postoperative adjunctive pain control was provided by IV-PCA hydromorphone and later transitioned to oral oxycodone/acetaminophen.

All patients received daily $40 \mathrm{mg}$ subcutaneous injections of enoxaparin (Lovenox ${ }^{\circledR}$, Sanofi-Aventis), starting no sooner than $12 \mathrm{~h}$ after surgery with the last dose administered at 9AM on POD 2. In patients undergoing major hepatic resection, the coagulation profile was followed closely and LMWH was held if the PT was prolonged. Fresh frozen plasma was given if the PT was greater than 17 in the first 24 hours. All catheters were removed after 9AM on POD 3 after verifying a normal coagulation profile and platelet count. All non-steroidal anti-inflammatory drugs and anti-platelet drugs, such as aspirin or clopidogrel, were withheld during co-administered continuous epidural analgesia and LMWH. Nonpharmacological anti-thrombotic mechanical compression devices were used on all patients peri-operatively.
Data abstracted included age, ethnicity, sex, average length of stay, duration of epidural placement, and incidence of VTE events and epidural complications.

\section{Postoperative Complications Explained}

\subsection{Spinal Hematoma}

New onset back pain, progressive lower extremity numbness or weakness, bowel or bladder dysfunction, radiological signs of cord compression requiring decompression laminectomy [3].

\subsection{VTE}

Deep Vein Thrombosis (DVT): New onset lower extremity pain, swollen limb, and/or positive non-invasive venous studies utilizing Color Doppler Spectral Analysis and B-mode ultrasound imaging.

Pulmonary Embolism (PE): Acute onset of dyspnea, tachycardia, hypotension, increased CVP, and/or positive V/Q scan or chest CTA requiring pharmacologic therapy [3].

\section{Results}

\subsection{Operative Procedures}

Two-hundred and thirty-seven patients underwent major foregut surgeries in one of five categories: 74 patients underwent pancreatic surgery (31.2\%); 60 underwent complex biliary surgery (25.3\%); 59 underwent hepatic surgery (24.9\%); 39 underwent gastrointestinal surgery (16.5\%); 5 underwent splenic surgery (2.1\%). Patients were categorized based on the dominant surgical procedure.

Mean length of stay was recorded for all procedures: gastrointestinal surgery $13.5 \mathrm{~d}$, pancreatic surgery $13.2 \mathrm{~d}$, biliary surgery $10.3 \mathrm{~d}$, hepatic surgery 6.4d, splenic surgery $5.4 \mathrm{~d}$. Malignancy was the indication for surgery in $69.2 \%$ of all patients $(\mathrm{n}=164)$, including $81.4 \%$ of patients undergoing hepatic procedures $(n=48), 76.7 \%$ of patients undergoing biliary procedures $(n=46), 58.1 \%$ of

Table 1. Summary of results for all 237 patients.

\begin{tabular}{|c|c|c|c|c|c|c|c|c|c|}
\hline \multirow{2}{*}{$\begin{array}{l}\text { Foregut } \\
\text { procedure }\end{array}$} & \multirow{2}{*}{ Number of patients } & \multicolumn{2}{|c|}{ Mean age } & \multirow{2}{*}{$\begin{array}{c}\text { Mean length of stay } \\
\text { (days) }\end{array}$} & \multirow{2}{*}{$\begin{array}{l}\text { Epidural duration } \\
\text { (days) }\end{array}$} & \multirow{2}{*}{$\begin{array}{l}\text { Epidural } \\
\text { hematoma }\end{array}$} & \multirow{2}{*}{$\begin{array}{l}\text { Malignant } \\
\text { lesions }\end{array}$} & \multicolumn{2}{|c|}{$V T E^{*}$} \\
\hline & & Male & Female & & & & & $D V T^{\dagger}$ & $P E^{\ddagger}$ \\
\hline Gastrointestinal & 39 (16.5\%) & 67 & 63 & 13.5 & 3 & 0 & 24 (61.5\%) & 1 & 1 \\
\hline Pancreatic & $74(31.2 \%)$ & 64 & 56 & 13.2 & 3 & 0 & 43 (58.1\%) & 1 & 0 \\
\hline Hepatic & $59(24.9 \%)$ & 61 & 54 & 6.4 & 3 & 0 & 48 (81.4\%) & 1 & 0 \\
\hline Biliary & $60(25.3 \%)$ & 68 & 59 & 10.3 & 3 & 0 & $46(76.7 \%)$ & 1 & 0 \\
\hline Splenic & $5(2.1 \%)$ & 60 & 41 & 5.4 & 3 & 0 & $3(60.0 \%)$ & 0 & 0 \\
\hline Overall & 237 & 65 & 57 & 10.7 & 3 & 0 & $164(69.2 \%)$ & 4 & 1 \\
\hline
\end{tabular}

The four patients with DVT had bilateral lower extremities involved. ${ }^{*}$ VTE: venous thromboembolism; ${ }^{\dagger}$ DVT: deep venous thrombosis; ${ }^{\ddagger}$ PE: pulmonary embolism. 
patients undergoing pancreatic procedures $(n=43), 61.5 \%$ of patients undergoing gastrointestinal procedures ( $\mathrm{n}=$ 24 ), and $60 \%$ of patients undergoing splenic procedures $(n=3)$ (Table 1).

\subsection{Post-Operative Care and Complications}

All epidural catheters were placed preoperatively and removed on the third post-operative day. There were a total of five VTEs reported postoperatively consisting of four DVTs and one PE (Table 2). The first VTE complication was an acute bilateral DVT in a 79-year-old female, who underwent a common bile duct exicison, portal lymphadenectomy and a Roux-en-Y hepaticojejunostomy for a mid-bile duct cholangiocarcinoma. The thrombus was located in the peroneal vein in the right leg and the gastrocnemius vein in the left leg. The second VTE complication was an acute DVT in a 73-year-old male, who underwent pancreaticoduodenectomy for pancreatic cancer. The thrombus was located in the popliteal vein above the knee in the right leg and in the popliteal vein at the knee in the left leg. The third complication was an acute bilateral DVT in a 67-year-old female, who underwent a right hepatectomy for metastatic colorectal cancer. The thrombus was located in the popliteal vein at the knee in the right leg and the external iliac, common femoral, and popliteal veins in the left leg. The fourth VTE complication was an acute bilateral DVT in an 83year-old female, who underwent a subtotal duodenectomy and Roux-en-Y gastrojejunostomy for a duodenal adenoma with high-grade dysplasia. The thrombus was located in the common femoral and popliteal veins in the right leg and the posterior tibial vein in the left leg. Symptoms prompting initial investigation in all four patients included pain and swelling of bilateral lower extremities. The fifth complication was a PE in a 76-yearold female, who underwent a palliative Roux-en-Y gastrojejunostomy and choledochojejunostomy for metastatic and completely obstructing duodenal carcinoma. The patient presented with tachycardia and shortness of breath, prompting chest CT angiogram. All patients were treated with therapeutic enoxaparin and bridged to war- farin as per ACCP guidelines [19]. There were no spinal or epidural complications associated with epidural catheter use and no patients returned after discharge for hemorrhagic sequela (Table 1). Venous Doppler studies were not performed preoperatively to assess for the possibility that DVT may have existed preoperatively in this high risk population.

\section{Discussion}

The statistically significant benefits of epidural anesthesia/analgesia have been demonstrated in the literature for four major organ systems: cardiovascular, gastrointestinal, pulmonary, and renal [15]. Specifically, epidural anesthesia blocks the perioperative sympathetic activation associated with major surgery, causing a reduction in cardiac morbidity characterized by decreased myocardial infarction, blood loss, transfusion requirement, and DVT $[15,16]$. Sympathetic nerve block leaves unopposed parasympathetic innervation to the gut, resulting in reduction of postoperative ileus [15]. Thoracic epidural anesthesia/ analgesia with a local anesthetic and general anesthesia increases the functional residual capacity by $27 \%$ and shows an overall improvement in pulmonary outcome characterized by decreased pulmonary infection, pulmonary embolism, respiratory depression, and intubation time $[15,17,18]$. Epidural anesthesia/analgesia also protects kidney function by reducing the incidence of acute renal failure [4].

VTE is a frequent cause of mortality and morbidity among surgical patients [19]. Mukherjee et al conducted a retrospective analysis of 375,748 patients comparing VTE risk across eight major surgical procedures: bariatric surgery, colorectal surgery, esophagectomy, gastrectomy, hepatectomy, nephrectomy, pancreatectomy, and splenectomy [14]. The study demonstrated a $1.54 \%$ overall rate of VTE, with the lowest incidence present in patients undergoing bariatric surgery (0.35\%) [14]. VTE rates were higher in patients undergoing hepatectomy (1.76\%), splenectomy (2.36\%), gastrectomy (2.58\%), and pancreatectomy (2.91\%) [14]. Overall death rate increased from $3.97 \%$ to $13.34 \%$ in patients diagnosed with VTE [14].

Table 2. Summary of VTE complications.

\begin{tabular}{|c|c|c|c|c|c|}
\hline \multirow{2}{*}{ Age } & \multirow{2}{*}{ Sex } & \multirow{2}{*}{ Diagnosis } & \multirow{2}{*}{ Procedure } & \multicolumn{2}{|c|}{$V T E^{*}$} \\
\hline & & & & $D V T^{\dagger}$ & $P E^{\ddagger}$ \\
\hline 79 & $\mathrm{~F}$ & Cholangiocarcinoma & Roux-en-Y hepaticojejunostomy & 1 & 0 \\
\hline 73 & M & Pancreatic cancer & Pancreaticoduodenectomy & 1 & 0 \\
\hline 67 & $\mathrm{~F}$ & Metastatic colorectal cancer & Partial hepatectomy & 1 & 0 \\
\hline 83 & $\mathrm{~F}$ & Duodenal cancer & Subtotal duodenectomy \& Roux-en-Y gastrojejunostomy & 1 & 0 \\
\hline 76 & $\mathrm{~F}$ & Duodenal cancer & Roux-en-Y gastrojejunostomy \& choledochojejunostomy & 0 & 1 \\
\hline
\end{tabular}

The four patients with DVT had bilateral lower extremities involved. ${ }^{*}$ VTE: venous thromboembolism; ${ }^{\dagger}$ DVT: deep venous thrombosis; ${ }^{\ddagger} \mathrm{PE}$ : pulmonary embolism. 
Studies in general surgery patients comparing thromboprophylaxis using unfractionated heparin versus no thromboprophylaxis have demonstrated a significant reduction in the rate of deep vein thrombosis (DVT), symptomatic PE, fatal PE, and all-cause mortality [20]. The risk of symptomatic VTE and asymptomatic DVT is reduced by more than $60 \%$ in general surgery patients with the use of unfractionated heparin or low-molecular-weight heparin (LMWH), compared to no thromboprophylaxis [19]. A decreased risk of heparin-induced thrombocytopenia and once-daily dosing are examples of several clinical advantages of LMWH when compared to unfractionated heparin [19].

Cancer further increases the risk of VTE seven-fold [21]. Patients undergoing surgery for malignancy have at least twice the risk of postoperative DVT and more than three times the risk of fatal PE compared to non-cancer patients undergoing similar surgery [19]. As such, aggressive thromboprophylaxis, as recommended by the ACCP, is imperative in these patients [19]. LMWH is easy to administer, has predictive pharmacokinetics, does not require routine therapeutic monitoring, and is the foundation of VTE treatment and prophylaxis in cancer patients across most guidelines [22]. However, cancer is also an independent predictor of thromboprophylaxis failure and adequate thromboprophylaxis will not eliminate the risk of VTE [19].

The American College of Chest Physicians (ACCP) has established guidelines for the appropriate use of both mechanical and chemical thromboprophylaxis during surgical procedures [19]. Sporadic reports of neurologic complications, resulting from spinal hematomas, have created controversy regarding concomitant use of LMWH with epidural anesthesia. These concerns prompted the FDA to require a black box warning to be included with the package insert for LMWH urging extreme caution in the presence of an indwelling catheter [23-26]. In order to address this issue, the ACCP and the American Society of Regional Anesthesia and Pain Medicine (ASRA) published guidelines regarding the timing of LMWH and neuraxial anesthesia and the need for on-going neurologic monitoring wherever LMWH is used in combination with neuraxial anesthesia (Figure 1) [19,27].

Although rare and usually seen in patients with underly-

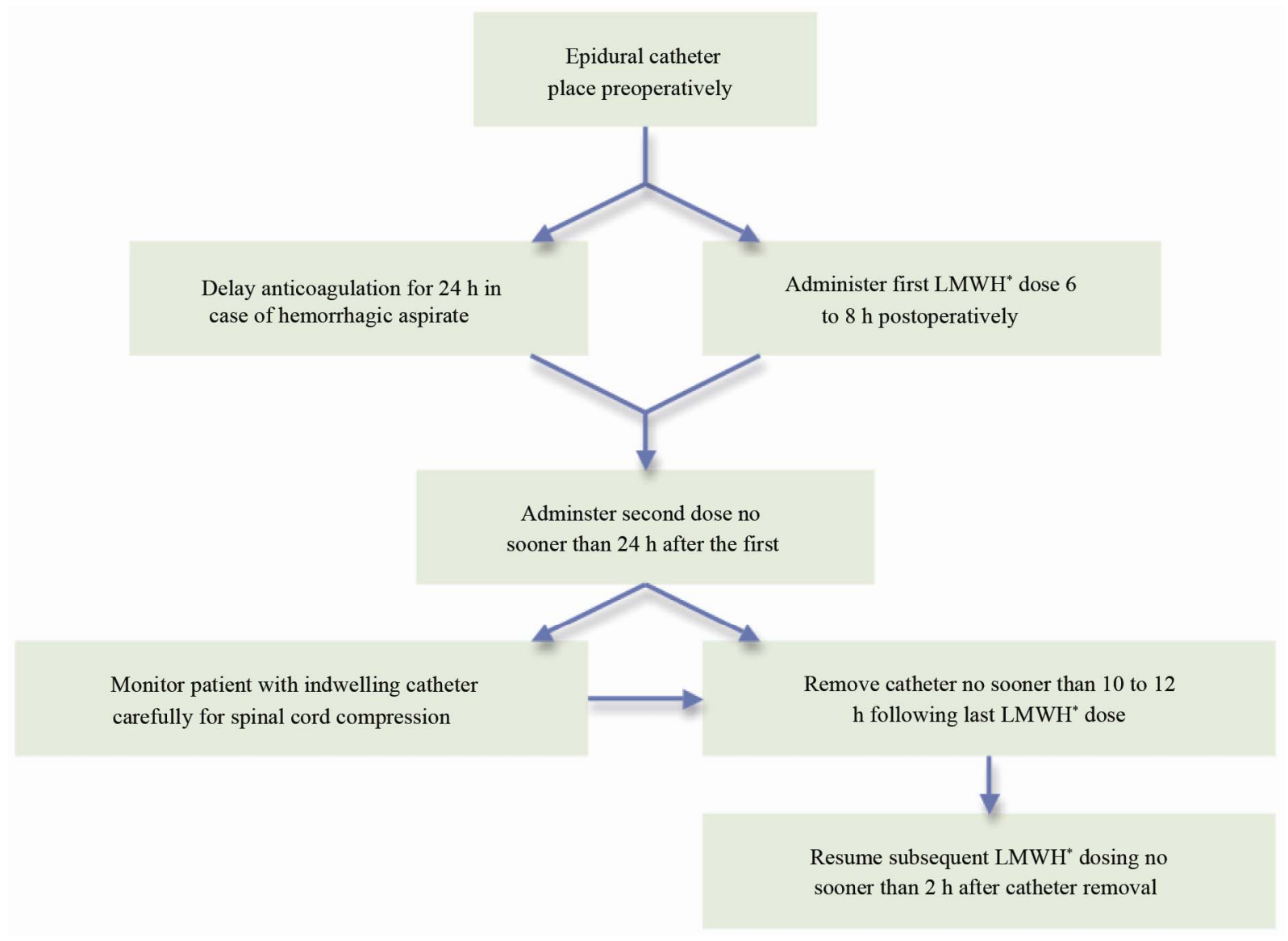

"LMWH: Low-molecular-weight heparin.

Figure 1. American society of regional anesthesia and pain medicine second consensus statement on neuraxial anesthesia and postoperative once-daily LMWH dosing [27]. 
ing coagulation abnormalities, spinal hematomas may lead to irreversible neurological injury and paraplegia [8, 23]. The ASRA and the ACCP guidelines on the use of neuraxial catheters with anticoagulation were developed in an effort to minimize the complication of spinal hematoma [19,27]. According to the 2002 ASRA consensus statement, safety is based on total daily dosage amount, timing of the first postoperative dose, and dosing schedule (Figure 1) [27]. The first LMWH dose should be administered 6 to $8 \mathrm{~h}$ postoperatively, the second dose should follow no sooner than $24 \mathrm{~h}$ after the first [27]. In the case of hemorrhagic aspirate during initial spinal needle placement, anticoagulation must be delayed for 24 $\mathrm{h}$ postoperatively [27]. Indwelling catheters should be removed no sooner than 10 to $12 \mathrm{~h}$ following the last LMWH dose, when anticoagulant effect is at a minimum [27]. Subsequent LMWH dosing should resume no sooner than $2 \mathrm{~h}$ after discontinuation of epidural catheter [27]. Finally, patients with continuous epidural analgesia and LMWH thromboprophylaxis should be carefully monitored for symptoms of spinal cord compression [27]. These include new onset back pain, progressive lowerextremity numbness or weakness, and bowel or bladder dysfunction [27]. The recommendations by the $8^{\text {th }}$ ACCP Concensus report are essentially identical to those proposed by the ASRA [19].

When these published guidelines are adhered to, a very low incidence of spinal hematomas has been reported. Several authors have reported an incidence of 1:150,000 after epidural anesthesia and 1:220,000 after spinal anesthesia [28-30]. Some authors, most notably Chelly et al, contend that strict guidelines concerning post-operative epidural catheter removal are unnecessary and have reported an 11-year complication-free experience [31,32]. In this study, patients were started on anticoagulation no sooner than 12 hours after surgery [32]. Lumbar catheters were removed on closing with no concern to the time of anticoagulant administration [32]. Patients were then monitored for spinal hematoma symptoms. A total of 6935 were performed in patients on thromboprophylaxis and no spinal hematomas were recorded [32].

Despite the aforementioned low overall incidence of spinal hematoma, several studies in living-related liver transplant donors have discouraged the use of epidural analgesia [11,12]. These papers suggest that major liver resection would adversely affect peri-operative coagulation profiles and increase the risk and development of epidural hematoma [11,12]. Choi et al. reported significant postoperative changes in the coagulation profile of 360 living liver donors consistent with the current literature [9]. Platelet count was lowest on POD 2 - 3, while PT peaked on POD 1 and a PTT was highest immediately after the surgery [9]. Of note, these patients received 4000 - 5000 IU of heparin intraoperatively, more than 4 hours after epidural catheter insertion, to prevent clotting in the graft following interruptions of the hepatic artery, hepatic vein, and portal vein [9]. Despite an unfavorable coagulation profile in the 242 living liver donors receiving epidural analgesia, no persistent neurological or epidural hematoma complications were reported. The mean duration of epidural catheter placement was 3.2 days (range, 0 - 6) [9].

Additional studies on the safety of epidurals in surgical procedures associated with high bleeding rates, such as cardiovascular surgery, have also begun to emerge. Ruppen et al performed an extensive systematic review of the literature demonstrating use of epidural catheters on anti-coagulated patients during vascular, cardiac, and thoracic surgery [2]. Analysis of twelve studies with 14,105 patients showed no cases of epidural hematoma in 5026 (35.6\%) patients undergoing vascular surgery, 4971 (35.2\%) cardiac surgical patients (coronary artery bypass graft surgeries), and 4108 (29.1\%) thoracic surgical patients [2]. All cardiac patients were fully heparinized, and 4054 of the 4108 thoracotomy patients (99\%) received 5000 IU of subcutaneous heparin two hours prior to surgery and every 12 hours while immobilized [2]. Among the three studies reporting on vascular surgery and extensive anticoagulation, including intraoperative systemic heparin, temporary neurologic injury consisting of transient monoplegia was reported in a total of 8 out of 13,422 patients $(0.06 \%)$ [2]. Eleven of the 12 studies evaluating the incidence of permanent neurological injury found no cases to report [2].

Two large multi-center randomized controlled trials were recently published, detailing the effect of epidural anesthesia/analgesia on outcome after major abdominal surgery [3,33]. The Veterans Affairs Cooperative Studies Program (VACS) compared 2 groups of patients undergoing colon, aortic, biliary, or gastric surgery [3]. While patients in group $1(n=495)$ received general anesthesia followed by systemic opioid analgesia. Patients in group 2 ( $n=489)$ received combined epidural/general anesthesia followed by epidural morphine for an unspecified time. These patients were monitored for new occurrence of lower back pain, leg weakness, urinary/fecal incontinence, and radiological signs of cord compression requiring decompression laminectomy. The study reported no cases of epidural hematoma in 489 patients undergoing surgery with epidural anesthesia/analgesia [3].

The Multicenter Australian Study of Epidural Anesthesia (MASTER) trial enrolled 915 high-risk patients who had undergone major abdominal surgery [33]. The most common procedures involved bowel, aortic aneurysm, biliary, and gastric surgery. Patients were randomized to either general anesthesia followed by systemic opioid treatment or combined epidural/general anesthesia followed by $72 \mathrm{~h}$ of postoperative epidural analgesia with 
local anesthetic and opioids. Only 225 of 418 (53.8\%) patients with epidural catheters adhered to the epidural analgesia protocol. The majority (183 of 193 (94.8\%)) of epidural catheters were removed prior to the $72 \mathrm{~h}$ protocol due to undocumented reasons (60), inadequate postoperative analgesia (42), accidental dislodgement (26), medical orders (16), generalized sepsis (9), hemodynamic instability (5), leaking catheter (5), leg weakness or numbness (4), no pain (4), death (3), lack of specialist nursing care or intensive-care bed (2), patient's request (2), operation changed (1), urinary retention (1), respiratory failure (1), block too high (1), and blocked catheter (1). Notably, despite the occurrence of leg weakness/numbness and urinary retention, the authors did not attribute any adverse events to the epidural catheter placement [33].

Although these studies comment on the safety of epidural anesthesia/analgesia in major abdominal surgery, they do not specify a uniform dosing schedule of LMWH or other heparanoids [3,33]. The ASRA and the ACCP recommendations stress careful monitoring of the total daily dose and the timing of the first and subsequent doses of LMWH with the timing and management of the epidural [19,27]. The current study evaluated 237 patients receiving epidural anesthesia and analgesia combined with LMWH, based on the ASRA and the ACCP recommendations. Uniform protocols were in place regarding the timing of LMWH administration with epidural catheter insertion and removal. Patients were evaluated on a daily basis for symptoms of spinal cord compression and VTE disease. Coagulation profiles were closely monitored in patients undergoing hepatic resection. There were no cases of epidural hematoma and a total of five VTEs (four DVTs and one PE). All incidences of VTEs occurred in cancer patients despite adequate anticoagulation as per ACCP guidelines. The five patients who developed VTEs were then treated with therapeutic enoxaparin followed by bridging to warfarin as per ACCP guidelines [19].

This study provides validation of the safety of continuous epidural catheter analgesia in patients receiving LMWH anticoagulation after major abdominal surgery. The limitations to the study are the relatively small sample size. Second, the detection of epidural hematomas and VTEs was based solely on symptomatic events. Asymptomatic epidural bleeds may have gone undiagnosed due to the lack of routine spinal imaging. Third, the results hold specifically to patients receiving epidural analgesia combined with enoxaparin and may not be applicable to other LMWHs.

\section{Conclusion}

In conclusion, concomitant use of LMWH and epidural anesthesia/analgesia under a uniform protocol is both safe and feasible in major abdominal surgery. This includes patients undergoing major liver resection, who may experience transient postoperative dysregulation of their coagulation profile. Particular detail must be paid to the timing of catheter insertion/removal relative to LMWH administration and patients must be monitored for any neurologic complications.

\section{REFERENCES}

[1] F. J. Singelyn, C. C. Verheyen, F. Piovella, H. K. Van Aken and N. Rosencher, "The Safety and Efficacy of Extended Thromboprophylaxis with Fondaparinux after Major Orthopedic Surgery of the Lower Limb with or without a Neuraxial or Deep Peripheral Nerve Catheter: The EXPERT Study,” Anesthesia \& Analgesia, Vol. 105, No. 6, 2007, pp. 1540-1547.

doi:10.1213/01.ane.0000287677.95626.60

[2] W. Ruppen, S. Derry, H. J. McQuay and R. A. Moore, "Incidence of Epidural Haematoma and Neurological Injury in Cardiovascular Patients with Epidural Analgesia/ Anaesthesia: Systematic Review and Meta-Analysis," BMC Anesthesiology, Vol. 6, 2006, p. 10. doi:10.1186/1471-2253-6-10

[3] W. Y. Park, J. S. Thompson and K. K. Lee, "Effect of Epidural Anesthesia and Analgesia on Perioperative Outcome: A Randomized, Controlled Veterans Affairs Cooperative Study," Annals of Surgery, Vol. 234, No. 4, 2001, pp. 560-571.

doi:10.1097/00000658-200110000-00015

[4] A. Rodgers, N. Walker, S. Schug, A. McKee, H. Kehlet, A. van Zundert, D. Sage, M. Futter, G. Saville, T. Clark and S. MacMahon, "Reduction of Postoperative Mortality and Morbidity with Epidural or Spinal Anesthesia: Results from Overview of Randomised Trials," British Medical Journal, Vol. 321, No. 7275, 2000, pp. 14931497. doi:10.1136/bmj.321.7275.1493

[5] S. J. Dolin, J. N. Cashman and J. M. Bland, "Effectiveness of Acute Postoperative Pain Management: I. Evidence from Published Data,” British Journal of Anaesthesia, Vol. 89, No. 3, 2002, pp. 409-423. doi:10.1093/bja/aef207

[6] K. J. Tuman, R. J. McCarthy, R. J. March, G. A. DeLaria, R. V. Patel and A. D. Ivankovich, "Effects of Epidural Anesthesia and Analgesia on Coagulation and Outcome after Major Vascular Surgery,” Anesthesia \& Analgesia, Vol. 73, No. 6, 1991, pp. 696-704. doi:10.1213/00000539-199112000-00005

[7] M. H. Prins and J. Hirsh, “A Comparison of General Anaesthesia and Regional Anaesthesia as a Risk Factor for Deep Vein Thrombosis Following Hip Surgery: A Critical Review," Thrombosis and Haemostasis, Vol. 64, No. 4, 1990, pp. 497-500.

[8] E. P. Vandermeulen, H. Van Aken and J. Vermylen, “Anticoagulants and Spinal-Epidural Anesthesia,” Anesthesia \& Analgesia, Vol. 79, No. 6, 1994, pp. 1165-1177. doi:10.1213/00000539-199412000-00024

[9] S. J. Choi, M. S. Gwak and J. S. Ko, "The Changes in 
Coagulation Profile and Epidural Catheter Safety for Living Liver Donors: A Report on 6 Years of Our Experience," Liver Transplantation, Vol. 13, No. 1, 2007, pp. 62-70. doi:10.1002/lt.20933

[10] R. Shontz, V. Karupathy, R. Temple and T. J. Brennan, "Prevalence and Risk Factors Predisposing to Coagulopathy in Patients Receiving Epidural Analgesia for Hepatic Surgery," Regional Anesthesia and Pain Medicine, Vol. 34, No. 4, 2009, pp. 308-311. doi:10.1097/AAP.0b013e3181ac7d00

[11] D. S. Beebe, R. Carr, V. Komanduri, A. Humar, R. Gruessner and K. G. Belani, "Living Liver Donor Surgery: Report of Initial Anesthesia Experience,” Journal of Clinical Anesthesia, Vol. 12, No. 2, 2000, pp. 157-161. doi:10.1016/S0952-8180(00)00114-8

[12] G. Cammu, R. Troisi and O. Cuomo, "Anaesthetic Management and Outcome in Right-Lobe Living Liver-Donor Surgery,” European Journal of Anaesthesiology, Vol. 19, No. 2, 2002, pp. 93-98. doi:10.1017/S0265021502000169

[13] W. Ruppen, S. Derry, H. McQuay and R. A. Moore, "Incidence of Epidural Hematoma, Infection, and Neurologic Injury in Obstetric Patients with Epidural Analgesia/ Anesthesia,” Anesthesiology, Vol. 105, No. 2, 2006, pp. 394-399. doi:10.1097/00000542-200608000-00023

[14] D. Mukherjee, A. O. Lidor, K. M. Chu, S. L. Gearhart, E. R. Haut and D. C. Chang, "Postoperative Venous Thromboembolism Rates Vary Significantly after Different Types of Major Abdominal Operations," Journal of Gastrointestinal Surgery, Vol. 12, No. 11, 2008, pp. 20152022. doi:10.1007/s11605-008-0600-1

[15] R. J. Moraca, D. G. Sheldon and R. C. Thirlby, “The Role of Epidural Anesthesia and Analgesia in Surgical Practice,” Annals of Surgery, Vol. 238, No. 5, 2003, pp. 663673. doi:10.1097/01.sla.0000094300.36689.ad

[16] W. S. Beattie, N. H. Badner and P. Choi, "Epidural Analgesia Reduces Postoperative Myocardial Infarction: A Meta-Analysis,” Anesthesia \& Analgesia, Vol. 93, No. 4, 2001, pp. 853-858. doi:10.1097/00000539-200110000-00010

[17] W. M. Wahaba, H. F. Don and D. B. Craig, "Post Operative Epidural Analgesia: Effects on Lung Volume,” Canadian Anaesthetists' Society Journal, Vol. 22, No. 4, 1975, pp. 519-527. doi:10.1007/BF03004868

[18] C. Jayr, H. Thomas, A. Rey, F. Farhat, P. Lasser and J. L. Bourgain, "Postoperative Pulmonary Complications: Epidural Analgesia Using Bupivacaine and Opioids versus Parenteral Opioids,” Anesthesiology, Vol. 78, No. 4, 1993, pp. 666-676. doi:10.1097/00000542-199304000-00009

[19] W. H. Geerts, G. F. Pineo, J. A. Heit, D. Bergqvist, M. R. Lassen, C. W. Colwell and J. G. Ray, "Prevention of Venous Thromboembolism: The Seventh ACCP Conference on Antithrombotic and Thrombolytic Therapy," Chest, Vol. 126, No. 3, 2004, pp. 388S-400S. doi:10.1378/chest.126.3_suppl.338S

[20] R. Collins, A. Scrimgeour and S. Yusuf, "Reduction in Fatal Pulmonary Embolism and Venous Thrombosis by Perioperative Administration of Subcutaneous Heparin:
Overview of Results of Randomized Trials in General, Orthopedic, and Urologic Surgery," New England Journal of Medicine, Vol. 318, No. 18, 1988, pp. 1162-1173. doi:10.1056/NEJM198805053181805

[21] J. W. Blom, C. J. Doggen, S. Osanto and F. R. Rosendaal, "Malignancies, Prothrombotic Mutations, and the Risk of Venous Thrombosis," Journal of the American Medical Association, Vol. 293, No. 6, 2005, pp. 715-722. doi:10.1001/jama.293.6.715

[22] L. J. Petersen, “Anticoagulation Therapy for Prevention and Treatment of Venous Thromboembolic Events in Cancer Patients: A Review of Current Guidelines," Cancer Treatment Reviews, Vol. 35, No. 8, 2009, pp. 754-764. doi:10.1016/j.ctrv.2009.08.009

[23] M. M. Lumpkin, “FDA Public Health Advisory,” Anesthesiology, Vol. 88, No. 2, 1998, pp. 27A-28A. doi:10.1097/00000542-199802000-00001

[24] T. T. Horlocker and J. A. Hert, "Low Molecular Weight Heparin: Biochemistry, Pharmacology, Perioperative Prophylaxis Regimens, and Guidelines for Regional Anesthetic Management,” Anesthesia \& Analgesia, Vol. 85, No. 4, 1997, pp. 874-875.

doi:10.1213/00000539-199710000-00031

[25] T. T. Horlocker and D. J. Wedel, "Neuraxial Block and Low-Molecular-Weight Heparin: Balancing Perioperative Analgesia and Thromboprophylaxis," Regional Anesthesia and Pain Medicine, Vol. 23, No. 6, Suppl. 2, 1998, pp. 164-177. doi:10.1016/S1098-7339(98)90143-2

[26] V. Moen, N. Dahlgren and L. Irestedt, "Severe Neurological Complications after Central Neuraxial Blockades in Sweden 1990-1999,” Anesthesiology, Vol. 101, No. 4, 2004, pp. 950-959. doi:10.1097/00000542-200410000-00021

[27] T. T. Horlocker, D. J. Wedel, H. Benzon, D. L. Brown, F. K. Enneking, J. A. Heit, M. R. Mulroy, R. W. Rosenquist, J. Rowlingson, M. Tryba and C. S. Yuan, "Regional Anesthesia in the Anticoagulated Patient: Defining the Risks (The Second ASRA Consensus Conference on Neuraxial Anesthesia and Anticoagulation)," Regional Anesthesia and Pain Medicine, Vol. 28, No. 3, 2003, pp. 172-197. doi:10.1053/rapm.2003.50046

[28] N. L. K. Tam, C. Pac-Soo and P. M. Pretorius, "Epidural Haematoma after a Combined Spinal-Epidural Anaesthetic in a Patient Treated with Clopidogrel and Dalteparin,” British Journal of Anaesthesia, Vol. 96, No. 2, 2006, pp. 262-265. doi:10.1093/bja/aei297

[29] M. Tryba, "Epidural Regional Anesthesia and Low Molecular Heparin: Pro (in German)," Anasthesiol Intensivmed Notfallme Schmerzther, Vol. 28, No. 3, 1993, pp. 179-181. doi:10.1055/s-2007-998902

[30] J. Castillo, X. Santiveri, F. Escolano, J. Castaño, C. Gomar and J. Canet-Sabaté, "Incidencia de Hematomas Espinales con Compresión Medular Relacionados con Anestesias Neuroaxiales en Cataluña,” Revista Española de Anestesiología y Reanimación, Vol. 54, 2007, pp. 591595.

[31] J. E. Chelly, "Do We Really Need an Interval between Administering Fondaparinux and Removing a Lumbar Plexus Catheter?” Anesthesia \& Analgesia, Vol. 108, No. 
2, 2009, pp. 670-671.

doi:10.1213/ane.0b013e31818eca57

[32] J. E. Chelly and D. Schilling, "Thromboprophylaxis and Peripheral Nerve Blocks in Patients Undergoing Joint Arthroplasty and Hip Fracture Surgery," Journal of Arthoplasty, Vol. 23, No. 3, 2008, pp. 350-354. doi:10.1016/j.arth.2007.05.045
[33] J. R. Rigg, K. Jamrozik, P. S. Myles, B. S. Silbert, P. J. Peyton, R. W. Parsons, K. S. Collins and MASTER Anaethesia Trial Study Group, "Epidural Anesthesia and Analgesia and Outcome of Major Surgery: A Randomized Trial,” Lancet, Vol. 359, No. 9314, 2002, pp. 12761282. doi:10.1016/S0140-6736(02)08266-1 